\title{
44612 - ANESTHETIC MANAGEMENT OF A PARTURIENT WITH ORNITHINE TRANSCARBAMYLASE DEFICIENCY
}

\author{
Mark Lewis, Department of Anesthesia, London Health Science Centre, London, ON, \\ Canada; \\ Indu Singh, University of Western Ontario; \\ Chitra Prasad, University of Western Ontario; \\ T Rupar, University of Western Ontario; \\ P Jones, University of Western Ontario;
}

PURPOSE: Ornithine Transcarbamylase Deficency (OTCD) is a rare genetic condition that predisposes the patient to acute states of hyperammonemia. Acute decompensation, including increased intracranial pressure (ICP) has been documented in patients with late onset OTCD (1). Precipitating events in these patients include stressful circumstances such as surgery and the peripartum period $(2,3)$. We discuss the anesthetic management of a parturient with known late onset OTCD.

CLINICAL FEATURES: Consent was obtained from the patient for publication of this report. A 30 year-old primigravida with OTCD presented to our institution at 40 weeks with spontaneous rupture of membranes. Recent diagnosis of late onset OTCD had been made through family history and molecular analysis. Previous consultation at our institution's Metabolic Disease Clinic revealed normal ranges of serum ammonia, plasma amino acids and urine orotic acid. After clinical signs of increased ICP were ruled out, epidural analgesia was provided to decrease the stress of labour. A continuous infusion of Bupivicaine $0.125 \%$ and Fentanyl $2 \mathrm{ug} / \mathrm{ml}$ provided satisfactory labour analgesia. Serial laboratory investigations including serum ammonia, blood gases and electrolytes were gathered during admission. Ammonia levels ranged from $<9$ to $25 \mathrm{umol} / \mathrm{L}$. Other laboratory data were within normal limits. Infusions of sodium benzoate and sodium phenylacetate were available in case of hyperammonemia. A 10\% dextrose intravenous solution was infused to decrease catabolism and ammonia production. On the second day of admission the patient required Caesarian delivery secondary to poor progression of labour. This was performed successfully under epidural anesthesia with $2 \%$ lidocaine with epinephrine and preservative free morphine. A healthy male baby was born without incident. The patient progressed through an uneventful course in hospital. After a 48-hour observation period, the patient and child were discharged from hospital with scheduled follow-up in the Metabolic disease clinic.

CONCLUSIONS: This is the first English language report in adult anesthesia literature on the management of a parturient with OTCD. Collaboration is required between Anesthesia, Obstetrical and Metabolic Disease teams in the peripartum period. Epidural anesthesia may be desirable in these patients to blunt the stress response to labour. A cautious approach to neuraxial blockade is advised in patients with suspected hyperammonemia, as increased ICP may be present. Treatment of symptomatic patients ranges from ammonia scavengers to haemodialysis. Other goals of care include avoidance of ammonia-producing medications such as 
dimenhydrinate and corticosteroids, as well as close observation of patient and newborn in the postpartum period.

REFERENCES: 1. Intensive Care Med 2001 27:1962 2. J Inherit Metab Dis 2005 28:237-240

3. Eur J Paed Neuro 2003 7:115-121 